\title{
DESIGNING THE DESIGN PROCESS FOR EARLY PROBLEM DISCOVERY FOR A COMPLEX AERONAUTICS SYSTEMS CHALLENGE
}

\author{
Moore, Rachel Meredith (2); \\ McGowan, Anna-Maria Rivas (1); \\ Jeyachandran, Nathaneal (2); \\ Bond, Kathleen H. (2); \\ Williams, Daniel (1); \\ Cannon, David (3); \\ Rowan, Cortney T. (2) \\ 1: NASA Langley Research Center; \\ 2: Accenture; \\ 3: NASA Langley Postdoc, USRA
}

\begin{abstract}
The earliest stage in the innovation lifecycle, problem formulation, is crucial for setting direction in an innovation effort. When faced with an interesting problem, engineers commonly assume the approximate solution area and focus on ideating innovative solutions. However, in this project, NASA and their contracted partner, Accenture, collaboratively conducted problem discovery to ensure that solutioning efforts were focused on the right problems, for the right users, and addressing the most critical needs - in this case, exploring weather tolerant operations (WTO) to further urban air mobility (UAM) - known as UAM WTO. The project team leveraged generative, qualitative methods to understand the ecosystem, players, and where challenges in the industry are inhibiting development. The complexity of the problem area required that the team constantly observe and iterate on problem discovery, effectively "designing the design process." This paper discusses the approach, methodologies, and selected results, including significant insights on the application of early-stage design methodologies to a complex, system-level problem.
\end{abstract}

Keywords: Design process, Research methodologies and methods, Multi- / Cross- / Trans-disciplinary processes

\section{Contact:}

Moore, Rachel M

Accenture

Industry X

United States of America

rachel.a.moore@accenture.com

Cite this article: Moore, R. M., McGowan, A.-M. R., Jeyachandran, N., Bond, K. H., Williams, D., Cannon, D., Rowan, C. T. (2021) 'Designing the Design Process for Early Problem Discovery for a Complex Aeronautics Systems Challenge', in Proceedings of the International Conference on Engineering Design (ICED21), Gothenburg, Sweden, 16-20 August 2021. DOI:10.1017/pds.2021.116 


\section{INTRODUCTION}

NASA's Aeronautics Research Mission Directorate (ARMD) has been transforming aviation for over 100 years. From X-planes to new materials and new operational scenarios, NASA has pursued innovation in nearly every aspect of flight. "Every U.S. commercial aircraft and U.S. air traffic control tower has NASA-developed technology on board that helps improve efficiency and maintain safety" (NASA Aeronautics, Website). Beyond flight, as new, non-traditional users, manufacturers, and service providers shape emerging aviation markets, they also create opportunities to change society (NASA AAM, Website). To encourage this positive change and anticipate the aviation community's needs, NASA's gaze is fixed 25+ years into the future. One area that has attracted NASA's attention is Urban Air Mobility (UAM), where operations in any weather (more simply, Weather Tolerant Operations or WTO) can potentially enable transformative opportunities for several industries.

To explore and better understand the potential opportunities and related needs of the UAM WTO topic area, ARMD's Convergent Aeronautics Solution (CAS) project, part of the Transformative Aeronautics Concepts program (TACP), initiated a problem and market exploration effort. Accenture Federal Services, heretofore referred to as 'Accenture,' was competitively selected as the contractor. Together, the NASA and Accenture team partnered to better understand key problem areas in UAM WTO where NASA may conduct future research with a focus on innovation - specifically innovation that may have transformative impacts on the aviation industry and beyond. In this paper, we will discuss highlights and lessons from the rigorous design research process developed and used for UAM WTO effort. The design process itself was carefully designed to address specific needs for the topic area, for transformative innovation, and for NASA.

Beyond identifying important problem areas, NASA had an additional goal: assess how effective the innovative design process used can work at scale for CAS and enable it to respond quickly to change. For this collaborative effort, this meant completing the problem and market exploration effort in 14 weeks. While this goal was placed on and executed by Accenture, the rationale for the goal was a reflective one for NASA. Large organizations, particularly large government organizations, can unintentionally self-impose barriers to innovation and be slower responding to industrial change than desired. NASA continues to strive to be responsive to disruptions in aviation; thus challenging itself to learn faster, more effective ways to innovate. The CAS project intentionally seeks to learn quick, transformative, cross-disciplinary, and cross-industry innovation methods. For this NASA-Accenture collaboration, we tested rapid, early-stage design research on a complex problem with global implications, doing so within a complex organization (NASA) that has global public-private reach. Key insights from this rapid design innovation effort are discussed in this paper.

\section{WHY SHIFT APPROACH}

At the core of the NASA-Accenture collaboration was a shift in NASA's focus within the innovation lifecycle. Rather than first concentrating on solutions, time was invested in the earliest stages of the innovation process where problems are explored, needs are identified, and the most important systemlevel questions are defined. (Baer, 2013) This shift was somewhat unconventional for the worldrenown problem solvers at NASA. Whereas NASA's research, development, and engineering are known worldwide for bringing solutions to daunting, engineering challenges, the goal of this effort was to focus instead on the earliest stage of innovation: problem discovery.

Pursuing a new approach to problem discovery in the CAS project presented an opportunity to both observe the real-world effectiveness of methods and simultaneous real-time feedback from a those at NASA who would be expected to implement the process, should it become integral to the processes within the CAS project. The next section outlines the methodologies used and why they were selected to facilitate the shift to need-led problem discovery and definition, Section 3 outlines the rationale for modifications, and lessons learned from the team are reflected in Section 4. 


\subsection{Importance of framing to facilitate shift}

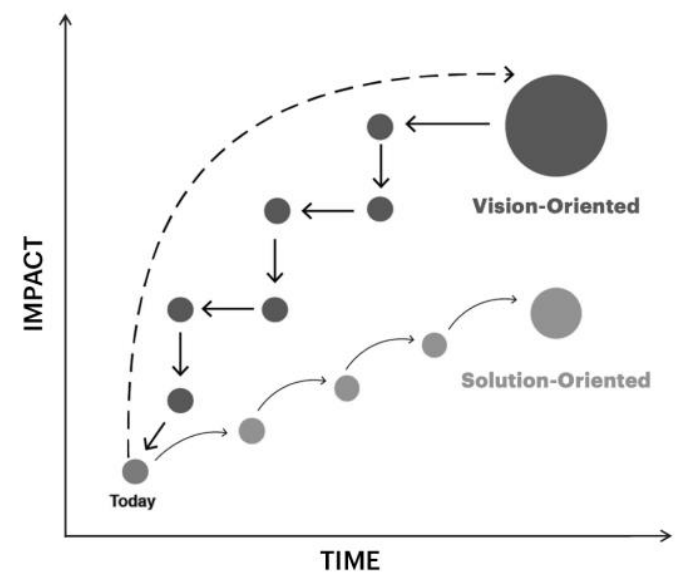

Figure 1. The backcasting model

The design team adopted several methods to shift the combined effort's focus to problem discovery. One critical framework was the Backcasting Model, as shown in Figure 1. With roots in urban planning, Backcasting is a vision-led approach that defines the desired future-state vision first, then considers different solutions paths to achieve the future state (Holmberg, 2000). This contrasts with a solution-focused approach, which typically takes incremental steps based on projected solutions. The Backcasting model shifts mindset from "What can we do?" (prompting incremental change) to "What do we need to do?". Shifting to the latter releases limiting constraints to foster greater innovation.

In this exploration, the Backcasting Model was foundational to framing a critical step in problem discovery and definition: prioritization of efforts. As exploration was planned, the team conducted a series of activities to generate potential future states, or visions. These prototype future states helped to prioritize stakeholder groups for deeper research in order to begin to generate the "backcast" steps.

Another component of this mindset shift from solution to problem was adjusting how the research question was formulated. For example, in a solution-focused approach, problem exploration focuses on identifying requirements for a presumed solution space. In this case, a representative research question would be, "How might we improve the use of [a specific solution] for UAM WTO?" In contrast, a problem-focused approach endeavours to be solution agnostic; instead, it focuses on identifying needs, from which a problem space is defined. With this approach, a representative research question would be, "How might we [solve this problem] for WTO?" In the second example, the question begets a wider range of potential solutions, more diverse teams, and higher impact outcomes.(Schön, 2011) This vision-led step toward problem discovery and definition resonated with the combined team, as noted in section 4.

Another reason for using both Backcasting and the question formulation exercises is that they are generative in nature. Generative methods are exploratory and challenge-focused to help gain a broader and better understanding of the problem (Ellamil, 2012). Once a level of understanding is established, later phases of work leverage often focus on more evaluative methods for measuring a proposed concept or problem area. Early in the effort, the team used highly generative, qualitative activities to draw out unmet needs and large focus groups to discern industry barriers. As potential problem areas surfaced out of the generative research, evaluative testing was used to test the validity of the more specific recommendations.

\subsection{Value of Qualitative Research Methods}

At NASA, quantitative methods are much more common; however for this effort, it was recognized that both qualitative and quantitative methods bring value to the early stage of the innovation lifecycle. In early stages of exploration, such as with this effort, the objective is to focus on need-finding and problem discovery, rather than requirements definition. Therefore, qualitative methods are the correct lens in the exploration stage, when the aim is to uncover unmet and unarticulated needs (Liamputtong, 2020). 
There are considerable texts that provide extensive comparison between qualitative and quantitative approaches for different purposes. For the current effort, we summarize below a brief comparison that the NASA-Accenture team considered as methods were considered for use.

\section{Some Considerations with Qualitative Research Methods}

- Focus on the "Why"

- Assume the key parameters need to be discovered and clarified

- Focus on understanding the complexities of the system context using a holistic approach

- More open-ended, allowing for unplanned data in the process of uncovering the unknown

\section{Some Considerations with Quantitative Research Methods}

- Focus on the "What"

- Assume the key parameters are already known and need to be measured

- Focus on classification, counting, or measuring known parameters

- $\quad$ Can be more fixed

\subsection{Learning From Early-Stage Design Methodologies}

A key value of a problem-focused exploration is that the projects' progress follows the problem rather than a specific technical discipline, component, or solution. While there is significant value in following the latter directions in other research and engineering efforts, those paths would typically limit the ceiling of an innovation project's potential. By pursuing the system-level problem and its needs, unconventional solutions are free to arise from new, unexpected directions that do not follow the historic path. This way, teams of engineers can explore the problem space from new perspectives. (London, 2012) Generative and qualitative approaches focused on the problem are excellent for discovering unarticulated needs and connecting diverse opportunities before formulating potential solutions.

Yet the problem-focused path is not as linear nor stepwise as following a known technology development path. It is an iterative path of discovery that must be adapted as new, relevant information is identified. Indeed, a key difference with early-stage problem discovery is the amount of ambiguity in the exploration. Hitting a "dead end," or following a line of inquiry that does not ultimately yield applicable insights is still considered informative (such as learning that a group is not interested in the problem or portions of it). These dead ends suggest insights such as: this is not where important needs or customers are; the assumptions behind the original line of inquiry need to be reformulated; and there are alternative (ambiguous) considerations. To help sense when to continue a line of inquiry or pivot in the ambiguous early stages of innovation, it is helpful to include an objective perspective on the team, write clear research objectives, and define a process to identify when it is time to pivot. This project leveraged both diversity across the combined team and clearly defined research objectives to support confidence in the project's direction.

Fundamentally, qualitative design research methods must be both rigorous and flexible. This allows for the research to follow its objective, and to continue to ask the question in different ways until there is sufficient data to support a synthesized point of view. Examples of this dichotomy can be observed in the team's approach to individual deep-dive interviews. These interviews were structured through discussion guides and pre-defined research objectives. In application, however, the interviewer also follows the participant's lead, adopting their vernacular and making observations that define the participant's unique perspective. (Rubin, 2005) This data is synthesized across multiple interviews, identifying trends and themes to create a representative point of view. These explorations help to hone the research objectives. Ultimately the synthesized findings from all methods yield data-driven and problem-focused recommendations and next steps that are suited to the context of the organization at hand.

Throughout the program, the combined team conducted qualitative research activities to support needled insights for accelerating UAM WTO. These activities included one-on-one deep-dive interviews, subject specific workshops, use of personas to identify key parameters in the problem definition, and resonance testing (checking back with potential stakeholders to update our problem hypotheses). With each of these activities, the core team iteratively modified the methods to better suit a system level problem — such as UAM WTO - and respond to the feedback of the implementing team. Key learnings in "designing the design process" for a system-level problem are outlined in the following section. 


\section{DESIGNING THE DESIGN PROCESS}

For the UAM WTO topic area and NASA's unique mission, the traditional problem discovery/ definition research approach required some unique modifications. For each, Accenture and NASA collaborated to maintain the rigor and quality of the design research process, yet tailor it to exist, resonate, and be repeatable in NASA's context. In this section we will highlight a few specific examples of how the collaborative team designed the process to preserve the integrity of the research structure and outputs, while simultaneously addressing NASA's unique mission and the WTO problem area.

\subsection{Ecosystem Considerations and NASA's Mission}

Often in product design, several user groups are identified. There may be primary and secondary user groups that are expected to be the core of the customer base, though designers must still make deliberate design decisions to ensure any customer can safely and effectively use the final design. NASA's federal government mission broadens the customer analysis to consider a national and global perspective so that societies across the US are served. In addition, as aviation is an interconnected global system, UAM WTO also must include considerations for the connected global private and public customer base.

\subsubsection{Considering the Ecosystem}

NASA's mission embraces non-commercial goals around crucial civil infrastructure needs such as emergency services and public transportation. These areas have widespread but perhaps less tangible economic benefits. They target important human, societal, and national needs and opportunities that can improve quality of life. As such, traditional design considerations for users were expanded in this research effort to include not only users (such as shipping companies and medical supply operators), but also stakeholders (such as other government agencies and local business leaders), as well as an expansive number of beneficiaries. A deeply societal and "everyday citizen" view was also embraced in the analysis. For example, the NASA-Accenture team conducted a workshop on smart cities that included aviation and outside-of-aviation thought leaders. The team also conducted interviews with citizens with no technical expertise in aviation.

\subsubsection{Incorporating New Entrants and Dynamics}

NASA's desire for transformational and disruptive innovation pressed the team's market exploration further. Aware of the dynamically changing aviation industry, NASA introduced the focus on nontraditional, novel, and emerging markets and industries. These included parties with whom NASA has not traditionally engaged. In pursuit of that mission, Accenture tapped their network (private industry, Accenture Ventures) to participate in a workshop with venture capitalists, interview start-up leaders, and tour start-up facilities.

The groups from the Accenture network have a deep understanding of investment and how early-stage companies are approaching the UAM industry. This extended network connected the NASAAccenture core team with key players in the nascent industry. Engaging with these industry participants in deep-dive interviews and workshops allowed the NASA-Accenture team to form a data-driven perspective on barriers for industry due to investment, regulation, and availability of technology. This was a critical component in defining the potential areas where NASA can move the UAM WTO industry forward. In addition to understanding the perspective of early-stage industry players, the team also developed an understanding of investors and how NASA can increase the momentum of investment in the industry. 


\subsubsection{Ecosystem Insights}

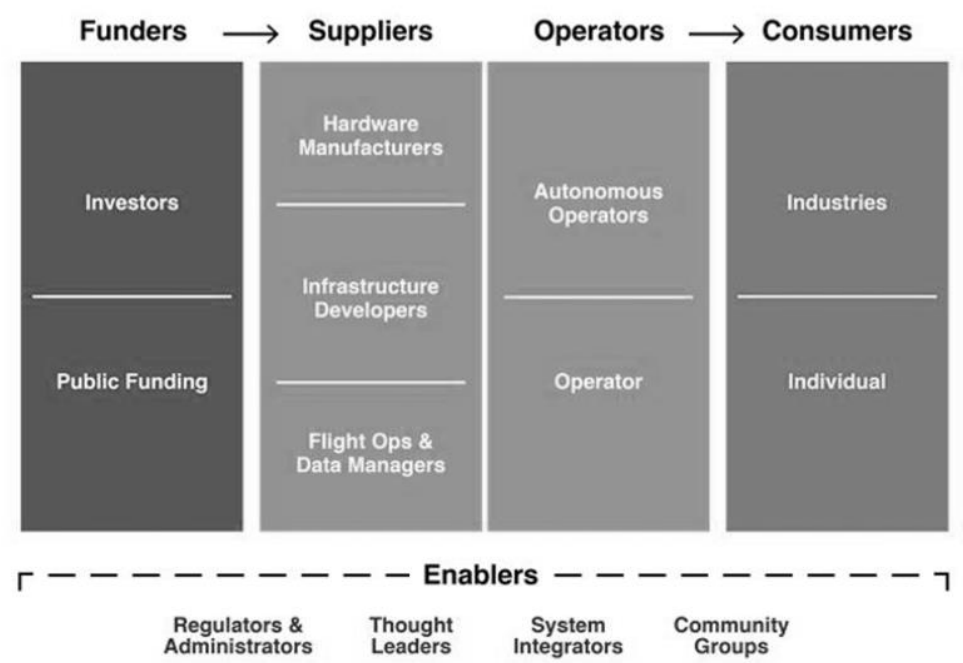

Figure 2. Example ecosystem

In the end, for this rapid design research project, the NASA-Accenture team conducted 27 deep-dive interviews with diverse USBs outside of NASA and 14 interviews with key stakeholders within NASA. The effort also included two workshops, each focused on a critical stakeholder group: venture capitalists and system integrators (defined as organizations that pull together disparate systems to create an offering within the UAM WTO industry). In the nascent UAM WTO industry, system integrators and members of the venture capital community are early adopters, making them a good proxy for identifying shared needs across the industry.

The combined output of the research led to the development of the following materials: personas representing groups of stakeholders, synthesized need states, defined bottlenecks, or "trapped value" in the industry, and materials around the structure and value transfer of the ecosystem. (Regan, 2019) A draft of one of the market ecosystems being analyzed is shown in Figure 2.

\subsection{Considerations for a Federal Government Lens}

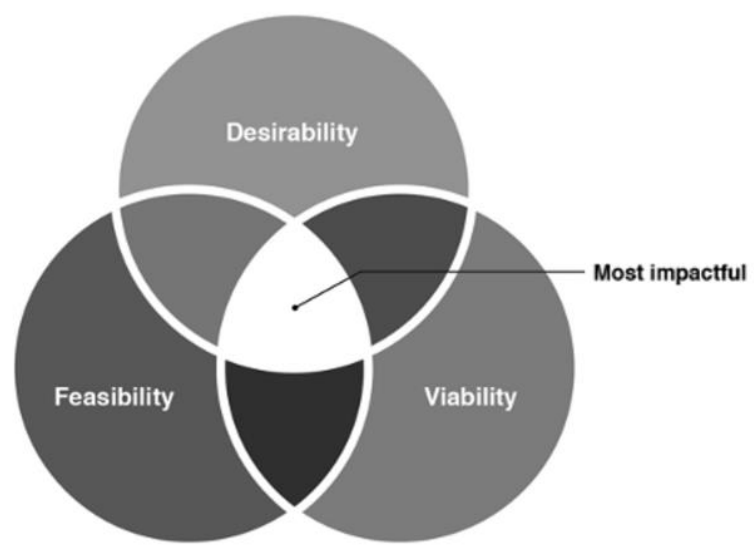

Figure 3. DVF model

As is always the case in design, what is "important" is subjective to the organization at hand. In this case, NASA wanted a federal government lens on a common design framework often referred to as Desirability, Viability, and Feasibility (DVF) shown in Figure 3.

The standard application of the DVF model considers three aspects of a concept. Desirability: Is the concept desired by the user? Viability: Is there a viable business path for this concept? Feasibility: Is this concept technically feasible? (Menold, 2016) For this effort, the DVF model had to consider the unique nature of NASA's mission in aeronautics. For NASA, the viability lens included policy, NASA organizational considerations, relationships with other agencies and countries, the economics related to civil infrastructures, and more. The desirability lens was expanded to include users, stakeholders, 
and beneficiaries (USBs), specifically where societal needs of relevance to aeronautics and potential value to USBs are considered.

The feasibility considerations were also unique. It is characteristic of NASA to confront hard, complex technical problems. So, for NASA, the difficulty of solving the technological problems was not seen as a barrier to constrain the early-stage problem discovery work and was taken off the table as an issue to consider at this stage in the design process. Rather, the team focused on the most important problems with the greatest potential impacts (problems that also addressed desirability and viability) during this early phase. Later, when solutions are ideated and explored, the team will certainly consider what is technically feasible. Thus, both the project team and the rest of their respective organizations prioritized working on the most impactful problems in UAM WTO and strongly desired the balanced perspective that the DVF model emphasizes. These considerations and the DVF model were helpful in prioritizing recommendations for NASA. Leading with needs, this more holistic prioritization acknowledges that the most technically feasible solution may not be the most impactful.

\subsection{NASA's Role}

In prioritizing the problems areas that warrant further exploration, the team also considered a variety of roles that NASA could execute, depending on several factors. This was essentially as aspect of the viability of the problem. Sourcing information from industry and NASA subject matter experts (SMEs), a few key themes arose from the research. First, NASA should operate in a role that leverages their unique capabilities to provide the most significant impact. In addition, NASA's engagement should be time bound, and the effort should sustain itself beyond NASA's investment. This "clear exit" will release NASA resources to move on to the next important problem.

An example of this prioritization would be a situation where an undertaking, while necessary, does not provide a clear path to profitability in the near-term. Imagine building a railroad — even though there is not a proportional return on investment, it enables significant societal benefits and opportunities for private industry to expand. Similar prioritization can be made in situations where NASA talent or facilities are needed as a "springboard" to address a challenge within the UAM WTO industry where critical, foundational problems need to be solved to enable profits in the longer term. Another example role for NASA is in "shepherding" key stakeholders into a collaborative interaction to define important technical directions that can spearhead new developments across multiple users.

\section{IMPLICATIONS AND OPPORTUNITIES}

For many years, human-centered design practices have been accepted as an important innovation method. In the study herein, these methods were expanded to include more complex, societal-level problems. This work has shown that beyond the products that sit on our kitchen counter, a design research perspective can bring also clarity to the needs of complex ecosystems, such as our cities, schools, and governments. (Ulrich, Eppinger, 2012) As mentioned previously, an important part of NASA's mission is to consider system-level problems and also to seek societal-level benefits.

\subsection{Innovating within NASA}

As change is the new steady-state, NASA continues to seek transformation within itself so that it can push the boundaries of flight to solve problems at the pace of industrial change. This effort brought together a diverse team to push the envelope: leveraging innovative problem and market exploration and analysis methods to identify the most significant opportunities for technological disruption. The collaborative effort described here was a targeted effort by NASA's CAS project to advance innovation within NASA aeronautics and challenge itself internally to learn improved ways to work and innovate - and to do so rapidly.

During this project, the NASA team had many insights regarding innovative work practices and how to potentially apply engineering design innovation to other projects. We share a few insights here with hope that we may encourage other large organizations in their pursuits of improving their internal innovation practices and processes.

- Gaining familiarity with and appreciation for the value of using several qualitative methods; understanding the rigor and expertise required and how and when these methods can be applied (as noted in section 2.2). Certainly, quantitative methods are much more the norm at NASA. 
- Noting the utility of dead ends (noted in section 2.3): They illustrated the tension between persistent exploration and speed in decision-making. Further, they reminded the team to focus on assessing the insights derived, rather than the natural tendency to view them solely as error.

- In a related perspective: welcoming iterations and pivots as opportunity more than miscalculation (as noted in 2.3)

- Reflectively acknowledging that most engineers are trained to think more evaluatively - the value of which cannot be overstated. However, we also recognize the need for generative approaches, as they are crucial to enable transformative innovation (as noted in section 2.1)

- Recognizing the rich value of the considerable professional diversity on the combined AccentureNASA team, with professional backgrounds that spanned many fields outside of aerospace. The collective insights from many domains brought invaluable perspective to the analysis and helped communicate outcomes in new, improved ways.

- Further expanding our appreciation for the non-commercial but important public service value that NASA can provide (as noted in 4.1).

- A perspective that balances desirability and viability is instrumental to enabling the systems-level transformations the team sought (as noted in 3.2)

- The design studio setting offered many tangible and intangible benefits such as: space to visually display and analyze disparate, unprocessed data and an environment that encourages informal and formal interdisciplinary interactions to enable opportunities for deeper and broader understanding.

While there were many other lessons learned, we close this section noting that both NASA and Accenture welcome collaboration with other organizations that seek to transform their operations internally. The challenges here are complex and thus often improved via learning from others. A summary of lessons learned from a previous CAS rapid design innovation effort can also be found in McGowan, et al (2017).

\subsection{Innovating at the Societal Level}

As we consider potential future efforts where we innovate at the societal level, the considerations and collateral impacts are wider. There is great potential for positive change, and great responsibility in understanding the impact of our efforts and creations. There is need for modifications to the standard process to consider broader impacts: How might our cities change as transportation moves from the ground to the air and hospitals and offices no longer need to be in the city center? How can we leverage new transportation systems to create more equity (in education for example)? What are our opportunities to improve everyday life by examining where and how we engage, and - equally important-where we don't?

Another societal-level example is: should UAM WTO become a reality, it would enable increased mobility of goods, services, and even people, even during extreme weather conditions. However, reaching these capabilities requires addressing key technical barriers like uncertainties in WTO missions (flight planning, micro-weather modeling, etc.). If solved, this knowledge base could unlock several avenues: empowering new entrants to focus the advancement of their capabilities for faster growth, supporting incumbents to optimize their capabilities more strategically, and allowing regulators to respond to expansions in industry more effectively.

Adopting a system and societal perspective also facilitated an understanding of the need for considering ecosystems holistically. It was observed that foundational WTO challenges are often addressed in a sparse and disconnected manner. Some of these foundational challenges (as an example, obtaining micro-weather data in a congested area) require multiple parties to coordinate and collaborate to address challenges that are unlikely to be solved by a single entity. In these areas, NASA can play a key role in shepherding the ecosystem to address foundational WTO challenges in a way that benefits many parts of the ecosystem. Done successfully, capabilities brought about via collaborative effort and industry alignment have potential for significant impact. For example, development of systems or enabling technology to address challenges in WTO may have potential to disrupt and improve markets adjacent to aviation where novel prospects for societal benefit may be exposed.

Once key issues to enable WTO are addressed and WTO becomes a reality, some industries and markets could change dramatically, offering new paths to deliver value to more people. Governments 
may be able to change the way services are provided, reaching more people. And, barriers for new entrants to enter certain markets could decrease once communities see the value. These types of considerations widen the impact of system-level design innovation in aeronautics to the societal level, offering potential benefits well beyond aviation.

\section{CONCLUDING REMARKS}

While design research is best known for how it can be used for products, this study demonstrated that innovative engineering design research can also be used to effectively tackle larger, more complex problems. NASA's vision in the CAS project is to bring transformational innovation in aeronautics to new arenas to make lasting positive impacts at a system-level. These efforts can also create important benefits at a societal level. During this rapid design innovation effort, the combined NASA-Accenture team deeply considered a wide-range of opportunities for impact.

The focus of the work was at the earliest stage of problem formulation. For the complex problem area examined (UAM WTO) and for NASA's mission, the innovative approaches used were rigorously drawn from many established design research principles. However, these design research principles were carefully designed and continuously shaped to effectively address the unique nature of a complex problem space and the NASA mission. In addition to addressing the topic area of UAM WTO, there were also important insights organizationally. The combined Accenture-NASA team learned from each other as innovative design research principles were judiciously adapted and applied. Today, NASA continues to apply many lessons learned from this work. We close this paper with an open invitation to the broader community for future collaborations on innovative methods to advance and evolve markets for the betterment of society through transformations in aeronautics.

\section{ACKNOWLEDGMENTS}

The authors wish to acknowledge the contribution and support of the broader NASA Accenture team. Specifically, Antoine Martin, Paul Barsamian, Jessica Hudson, Penelope Williams, Kenneth Jewell, Shannon Browning, Brian Ross, and Jennifer Close from Accenture. And, Andy Broeren, Kelley Hashemi, Francisco Sola, Amy Tarnowski, and Donna Gilchrist from NASA.

\section{REFERENCES}

Baer, M., Dirks, K.T. \& Nickerson, J.A., 2012. Microfoundations of strategic problem formulation. Strategic Management Journal, 34(2), pp.197-214.

Beckman, S.L. \& Barry, M., 2007. Innovation as a Learning Process: Embedding Design Thinking. California Management Review, 50(1), pp.25-56.

Ellamil, M. et al., 2012. Evaluative and generative modes of thought during the creative process. NeuroImage, 59(2), pp.1783-1794.

Fixson, S.K. \& Rao, J., 2014. Learning Emergent Strategies Through Design Thinking. Design Management Review, 25(1), pp.46-53.

Gipson, L., 2015. About Aeronautics Research Mission Directorate (ARMD). NASA. Available at: https://www.nasa.gov/aeroresearch/about-armd.

Gipson, L., 2019. Advanced Air Mobility (AAM). NASA. Available at: https://www.nasa.gov/aam.

Hey, J.H.G., Joyce, C.K. \& Beckman, S.L., 2007. Framing innovation: negotiating shared frames during early design phases. J. of Design Research, 6(1/2), p.79.

Holmberg, J. \& Robert, K.-H., 2000. Backcasting — a framework for strategic planning. International Journal of Sustainable Development \& World Ecology, 7(4), pp.291-308.

Kruger, C. \& Cross, N., 2006. Solution driven versus problem driven design: strategies and outcomes. Design Studies, 27(5), pp.527-548.

Liamputtong, P. \& Ezzy, D., 2009. Qualitative research methods, Oxford: Oxford University Press.

Liedtka, J., 2018. Why Design Thinking Works. Harvard Business Review. Available at: https://hbr.org/2018/09/why-design-thinking-works [Accessed 2020].

London, M., Sobel-Lojeski, K.A. \& Reilly, R.R., 2011. Leading Generative Groups. Human Resource Development Review, 11(1), pp.31-54.

McGowan, A.-M.R., Bakula, C. \& Castner, R.S., 2017. Lessons Learned from Applying Design Thinking in a NASA Rapid Design Study in Aeronautics. 58th AIAA/ASCE/AHS/ASC Structures, Structural Dynamics, and Materials Conference. 
Menold, J., Simpson, T.W. \& Jablokow, K.W., 2016. The Prototype for X (PFX) Framework: Assessing the Impact of PFX on Desirability, Feasibility, and Viability of End Designs. Volume 7: 28th International Conference on Design Theory and Methodology.

Regan, D., 2020. Trapped Value - What It Is And How To Unlock It: Accenture. WordPressBlog. Available at: http://www.accenture.com/us-en/blogs/voices-public-service/trapped-value-what-it-is-and-how-to-unlock-it [Accessed 2020].

Rubin, H.J. \& Rubin, I.S., 2005. Qualitative interviewing: the art of hearing data, London: SAGE.

Schön Donald A., 2016. The reflective practitioner: how professionals think in action, Abingdon, England: Routledge.

Seidel, V.P. \& Fixson, S.K., 2013. Adopting Design Thinking in Novice Multidisciplinary Teams: The Application and Limits of Design Methods and Reflexive Practices. Journal of Product Innovation Management, 30, pp.19-33.

Seow, O. et al., 2018. Design Signatures: Mapping Design Innovation Processes. Volume 7: 30th International Conference on Design Theory and Methodology.

Ulrich, K., 2012. Product Design and Development, New York: McGraw Hill / Irwin. 